\title{
Heavy-Fermions in $\mathrm{LiV}_{2} \mathrm{O}_{4}$ : Kondo-Compensation vs. Spin-Liquid Behavior?
}

\author{
H. Kaps, N. Büttgen, W. Trinkl, A. Loidl \\ Experimentalphysik V, Elektronische Korrelationen und Magnetismus, Institut für Physik, \\ Universität Augsburg, D-86135 Augsburg, Germany \\ M. Klemm, S. Horn \\ Experimentalphysik II, Institut für Physik, Universität Augsburg, D-86135 Augsburg, Germany
}

(November 13, 2018)

\begin{abstract}
${ }^{7} \mathrm{Li}$ NMR measurements were performed in the metallic spinel $\mathrm{LiV}_{2} \mathrm{O}_{4}$. The temperature dependencies of the line width, the Knight shift and the spin-lattice relaxation rate were investigated in the temperature range $30 \mathrm{mK}<T<280 \mathrm{~K}$. For temperatures $T<1 \mathrm{~K}$ we observe a spin-lattice relaxation rate which slows down exponentially. The NMR results can be explained by a spin-liquid behavior and the opening of a spin gap $\Delta_{S}$ of the order $0.6 \mathrm{~K}$.
\end{abstract}

75.20.Hr, 75.50.Lk, 76.60.Es, 76.60.-k

Recently, $\mathrm{LiV}_{2} \mathrm{O}_{4}$ gained considerable interest after reports of heavy-fermion formation at low temperatures [1.2. Based on heat capacity [2], spin-lattice relaxation [2]3], thermal expansion [1] and neutron scattering results [5] $\mathrm{LiV}_{2} \mathrm{O}_{4}$ was treated as a $d$-based heavy-fermion system [6], an interpretation which has been corroborated by band structure calculations [7].

$\mathrm{LiV}_{2} \mathrm{O}_{4}$ crystallizes in the fcc normal spinel structure and is characterized by vanadium ions in a $d^{1} / d^{2}$ mixed valence state. All band-structure calculations [7] 10] reveal the $t_{2 g}$ level close to the Fermi energy, separated into a lower $A_{1 g}$ and a higher $E_{g}$ orbital. It is emphasized that, in accordance with the Kondo-lattice interpretation, the $A_{1 g}$ electrons $\left(d^{1}, S=1 / 2\right)$ are localized, while the $E_{g}$ orbitals $\left(d^{0.5}\right)$ constitute the band states [7].

An alternative explanation of the spin-lattice relaxation rates in $\mathrm{LiV}_{2} \mathrm{O}_{4}$ has been provided within the framework of Moriya's theory [11] of ferromagnetic spin fluctuations [12] and quasielastic neutron scattering studies have provided experimental evidence that, in addition to the unconventional low-temperature properties, $\mathrm{LiV}_{2} \mathrm{O}_{4}$ undergoes a dramatic change of the spin-fluctuation spectrum. Below $40 \mathrm{~K}$ antiferromagnetic fluctuations dominate and the magnetic relaxation only weakly depends on momentum transfer, while at higher temperatures ferromagnetic correlations build up and the relaxation rates linearly increase on momentum transfer as observed in spin-fluctuation systems.

In this letter we report low-temperature NMR experiments extending to $30 \mathrm{mK}$. Temperature dependencies of line width, Knight shift and spin-lattice relaxation are reported at different measuring frequencies and external fields from $7.6 \mathrm{MHz} / 5 \mathrm{kOe}$ to $137 \mathrm{MHz} / 83 \mathrm{kOe}$, respectively. The temperature dependence of the spin-lattice relaxation rates convincingly demonstrates that in $\mathrm{LiV}_{2} \mathrm{O}_{4}$ the spin dynamics slows down exponentially but exhibits no static magnetic order. We conclude that $\mathrm{LiV}_{2} \mathrm{O}_{4}$ is similar to other frustrated spin-fluctuation systems like $\beta$-Mn [13] or Sc doped $\mathrm{YMn}_{2}$ [14], but in addition reveals the opening of a spin gap at low temperatures. We speculate about an unconventional magnetic ground state. It is unclear how this behavior can be reconciled with Kondo-compensation effects.

Polycrystalline samples of $\mathrm{LiV}_{2} \mathrm{O}_{4}$ were prepared by sintering a mixture of powders of $\mathrm{LiVO}_{3}$ and VO with slight excess of $\mathrm{LiVO}_{3}$ in order to compensate for Li evaporation. Platinum crucibles were used for reaction of the powders at $750{ }^{\circ} \mathrm{C}$ for 10 days. From EPR and magnetic susceptibility measurements, we estimated a number of $0.1 \% \mathrm{~V}$ defects. The NMR measurements were performed with a phase-coherent pulse spectrometer and spectra were obtained using field sweeps at constant frequencies $\omega / 2 \pi=7.6,17.3,72.7$ and $137 \mathrm{MHz}$. Cryogenic temperatures were provided by a ${ }^{3} \mathrm{He} /{ }^{4} \mathrm{He}$ dilution refrigerator with the NMR resonant circuit inside the mixing chamber. Probing the ${ }^{7} \mathrm{Li}$ nuclei (spin $I=3 / 2$, gyromagnetic ratio $\gamma=16.546 \mathrm{MHz} / \mathrm{T}$ ), we performed measurements of the line width $\delta$, the Knight shift $K$ and the spin-lattice relaxation rate $1 / T_{1}$. The spectra were collected using a conventional $\pi / 2-\tau_{D}-\pi$ spinecho sequence. The line width $\delta$ was deduced from the field-sweep spectra taking the full width at half maximum.

The spin-lattice relaxation rate $1 / T_{1}$ was determined from the inversion recovery of the spin-echo intensity. At low temperatures a stretched exponential relaxation behavior of the nuclear magnetization was observed as has been reported previously [15].

The spin-lattice relaxation rate $1 / T_{1}$ is generally described via the dynamical susceptibility $\operatorname{Im} \chi(q, \omega)$ as $[16$ ]

$$
T_{1}^{-1}=\frac{\gamma_{n}^{2} k_{B} T}{2 \mu_{B}^{2}} A_{\mathrm{hf}}^{2} \sum_{q} \frac{\operatorname{Im} \chi(q, \omega, T)}{\omega} .
$$


Here $A_{\mathrm{hf}}$ is the hyperfine coupling which is assumed to be isotropic and temperature independent. A Lorentzian-type function is used for the frequency dependence of the dynamical susceptibility

$$
\operatorname{Im} \chi(q, \omega)=\chi(q) \cdot \frac{\omega \Gamma}{\omega^{2}+\Gamma^{2}} .
$$

where $\Gamma$ is the magnetic relaxation rate which measures the characteristic energy of the spin-fluctuation spectrum. The prefactor $\chi(q)$ denotes the wave-vector dependent static susceptibility. In a first approach we neglect any $q$ dependence of the static susceptibility $\chi_{0}$.

Under the assumptions outlined above and including a 'metallic' Korringa term, the spin-lattice relaxation rate is given by

$$
T_{1}^{-1}=a \cdot T+b T \chi_{0} \cdot \frac{\Gamma(T)}{\omega_{0}^{2}+\Gamma^{2}(T)}
$$

where $b=\gamma_{n}^{2} k_{B} T A_{\mathrm{hf}}^{2} / 2 \mu_{B}^{2}$ is a constant and $\omega_{0}$ gives the Larmor frequency of the NMR experiment. The first term takes Korringa-type relaxations into account which stem from contributions of the band states. Using Eq.(3) the characteristic temperature dependence of the spin-lattice relaxation rate of heavy-fermion systems can be recovered assuming a Curie-Weiss like susceptibility, $\chi_{0}=C /\left(T+\alpha T^{*}\right)$ with $\alpha=\sqrt{2}$ and the characteristic Kondo temperature $T^{*}$ [17] and a magnetic relaxation rate that reveals a temperature dependence as $\Gamma(T)=\Gamma_{0}+\beta \sqrt{T}$ [18]. Under these simple assumptions the spin-lattice relaxation rate $1 / T_{1}$ reveals a cusp close to the characteristic temperature $T^{*}$ and a Korringa behavior with a highly enhanced slope for $T \ll T^{*}$. These are characteristic features which are observed experimentally in heavy-fermion compounds [19]. In $\mathrm{LiV}_{2} \mathrm{O}_{4}$ the temperature dependence of $1 / T_{1}(T)$ nicely resembles this behavior [2, 3, 12,15] and the square root dependence of the magnetic relaxation rate has been proven by neutron scattering experiments by Krimmel et al. [5].

Figure 1a shows the temperature dependence of the Knight shift at two measuring frequencies. The Knight shift provides a direct measure of the local static susceptibility. The cusp-like shape at approximately $30 \mathrm{~K}$ indicates the characteristic temperature $T^{*}$. The Knight shift is frequency/magnetic-field independent above $T^{*}$, but a significant dependence evolves below the temperature of the cusp maximum. For $T<T^{*}$ the Knight shift decreases approximately logarithmically and levels off at a constant value below $0.3 \mathrm{~K}$. Figure $1 \mathrm{~b}$ shows the temperature dependence of the line width $\delta$, again at two measuring frequencies. For both frequencies the line width continuously increases on decreasing temperatures and saturates below $1 \mathrm{~K}$. For the higher frequency and field the increase significantly is enhanced. The line width is dominated by an inhomogenous broadening due to local magnetic fields 3.15 .15 . The constant value at low temperatures signals that this internal fields become frozen and remain constant on the time scale of the experiment. However, below $0.1 \mathrm{~K}$ only a small fraction of nuclear spins contribute to the signal as it is clearly shown by the drastic decrease of the NMR intensity towards the lowest temperatures (see the inset in Fig.1).

The temperature dependence of the spin-lattice relaxation rate is shown in Fig. 2. Again, the maximum close to 30 $\mathrm{K}$ indicates the characteristic temperature $T^{*}$. This behavior nicely resembles the results found by Kondo et al. [2] . A Korringa-like behavior which has been determined by these authors in a temperature range from $1.5 \mathrm{~K}<T<6 \mathrm{~K}$ is indicated as solid line in Fig. 2. Our low temperature values of the spin-lattice relaxation rate at $72 \mathrm{MHz}$ remain slightly enhanced. This may be due to the fact that we analyzed the data assuming a stretched exponential recovery of the relaxation, as a pure exponential fit did not work especially at temperatures below $1 \mathrm{~K}$ [15. Astonishingly, at low measuring frequencies a clear cusp-shaped maximum appears at approximately $0.6 \mathrm{~K}$ which becomes almost suppressed at higher frequencies.

To study the anomalous low-temperature relaxation in more detail, we performed a series of experiments at different measuring frequencies and associated magnetic fields (Fig. 3a). Below $2 \mathrm{~K}$ the temperature dependence of $1 / T_{1}$ reveals a significant frequency dependence and the nuclear relaxation is strongly enhanced at low frequencies. This behavior clearly reveals a similar characteristic as the Li nuclear relaxation observed in $\mathrm{Li}$ doped $\mathrm{CuO}$ and $\mathrm{NiO}$ by Rigamonti and coworkers [20] which has been compared to the spin dynamics in cuprate superconductors. On the basis of this interpretation the cusp-like anomalies in Fig. 3a below $1 \mathrm{~K}$ signal the slowing down of spin fluctuations on a time scale given by the NMR experiments. We easily can incorporate this phenomenon in our model for correlated materials. Under the assumption of an exponentially increasing magnetic relaxation $\tilde{\Gamma}=\Gamma(T) \cdot \exp \left(-\Delta / k_{B} T\right)$ the spin-lattice relaxation rate $1 / T_{1}$ can be calculated using Eq.(3). Below $1 \mathrm{~K}$ the magnetic relaxation rate $\Gamma(T)$ is dominated by the exponential decrease, but it recovers the square root dependence at elevated temperatures. From the results of these calculations we obtain a rough estimate of the spin gap $\Delta$ to be of the order $1 \mathrm{~K}$ (Fig. $3 \mathrm{~b}$ ). The qualitative agreement is surprising having the very restrictive model assumptions in mind and especially neglecting the influence of the external magnetic field. On the basis of this results we conclude that, on decreasing temperatures 
and well below $1 \mathrm{~K}$ the spin fluctuations slow down exponentially and at the cusp maximum the measuring frequency directly corresponds to the relaxation rate. At temperatures $T<0.1 \mathrm{~K}$ the slow relaxation regime $(\Gamma \ll \omega)$ is reached. This observation of a slow spin dynamics down to the lowest temperature is in agreement with the $\mu$-SR results where the sample with the lowest impurity concentration revealed a slowing down of spin fluctuations with no signature of static freezing [2,21].

In Fig. 3c we show the low temperature spin-lattice relaxation rate in an Arrhenius representation to indicate the exponential increase of $1 / T_{1}(T)$ at low temperatures. We have subtracted the limiting low-temperature spin-lattice relaxation which is dominated by defect spins (see Inset in Fig. 1). For all frequencies we find an activated (solid lines) behavior corresponding to an effective spin gap of $\Delta_{S}=0.6 \mathrm{~K}$. The frequency dependence of $1 / T_{1}$ is weaker than $1 / \omega_{0}^{2}$ which is expected for the slow relaxation regime (see Eq.(3)). This fact points towards a broad distribution of relaxation rates [20].

How can these results be reconciled with a heavy-fermion picture and what is the intrinsic ground state of $\mathrm{LiV}_{2} \mathrm{O}_{4}$. Of course $\mathrm{LiV}_{2} \mathrm{O}_{4}$ is close to magnetic order. For example $5 \% \mathrm{Zn}$ doping induces spin-glass freezing at approximately $2.5 \mathrm{~K}$ 15. Certainly the same is true for prototypical heavy-fermion systems and we would like to recall that, depending on the exact Ce stoichiometry, $\mathrm{CeCu}_{2} \mathrm{Si}_{2}$ reveals superconductivity (S-phase) or magnetic order (A-phase). The regime of long-range magnetic order and $d$-wave superconductivity is separated by a phase which is dominated by slow magnetic fluctuations [22]. Based on a variety of different experiments, also the small-moment magnetism in $\mathrm{UPt}_{3}$ most probably is dynamic in origin. An oscillating spin-density wave, with a characteristic fluctuation rate of some $\mathrm{GHz}$, has been proposed to explain the results in this system [23].

All these facts reveal striking similarities with the experimental observations in $\mathrm{LiV}_{2} \mathrm{O}_{4}$. However, $\mathrm{LiV}_{2} \mathrm{O}_{4}$ also should be compared to canonical spin liquids. It is a frustrated magnet with the $\mathrm{V}$ ions forming a lattice of corner sharing tetrahedra. The Sommerfeld coefficient reaches $420 \mathrm{~mJ} /\left(\mathrm{mol} \mathrm{K}^{2}\right)$ [2] and the low-temperature magnetic relaxation rate is $0.5 \mathrm{meV}$ 5. The spin liquid $\mathrm{Sc}_{0.03} \mathrm{Y}_{0.97} \mathrm{Mn}_{2}$ reveals the same structural frustration, has a specific heat coefficient of $160 \mathrm{~mJ} /\left(\mathrm{mol} \mathrm{K}^{2}\right)$ and a magnetic relaxation rate of $8 \mathrm{meV}$. It's striking heavy-fermion like behavior has clearly been addressed [14. $\beta$-Mn is another geometrically frustrated magnet. Also this system reveals no static magnetic order and is characterized by a Sommerfeld coefficient $\gamma=70 \mathrm{~mJ} /\left(\mathrm{mol} \mathrm{K}^{2}\right)$ and a weakly temperature dependent magnetic relaxation rate $\Gamma \approx 20 \mathrm{meV}[13]$.

Astonishingly, spin liquids and heavy-fermions reveal very similar dynamical susceptibilities. In both cases low-lying and usually gapless magnetic excitations govern the dynamic susceptibility. However, the underlying physics seems to be rather different: In spin liquids long-range magnetic order is suppressed by topological magnetic frustration, soft magnetic excitations are enhanced favoring the formation of local singlets and the existence of an unconventional non-Néel magnetic ground state 24]. In heavy-fermion systems Kondo compensation yields an enhanced density of states at the Fermi energy driving the formation of heavy quasiparticles. Antiferromagnetic spin fluctuations screen the local moments.

What type of exotic ground state is established in $\mathrm{LiV}_{2} \mathrm{O}_{4}$ ? At temperatures $T>1 \mathrm{~K}$ the dynamical susceptibility is characteristic of a strongly correlated electron system. It is in this regime where spin-liquids and heavy-fermion systems behave similar. However, our results show that the magnetic relaxation $\Gamma(T)$ and consequently the spin-lattice relaxation rate $1 / T_{1}$ slow down exponentially indicating the opening of a spin gap of the order $0.6 \mathrm{~K}$. The gap could be due to dynamic singlet pairing. This interpretation is in accord with the 'cooperative paramagnet' which has been proposed by Villain [25] as a possible ground state of cubic spinels: The spins of each tetrahedron form antiparallel pairs, at least on a time scale large compared to the inverse NMR frequencies.

We believe that $\mathrm{LiV}_{2} \mathrm{O}_{4}$ is dominated by frustration effects rather than by moment compensation. Can we exclude a spin-glass transition? Exotic spin-glass behavior in systems without disorder has been proposed by Villain [25] who pointed out that canonical spin-glass behavior is unlikely to occure for spinels like $\mathrm{LiV}_{2} \mathrm{O}_{4}$. In addition, taking a Kondo-lattice temperature of $30 \mathrm{~K}$, it is hard to understand how random freezing of moments can appear well below $1 \mathrm{~K}$ with fully compensated moments. Also from a purely experimental point of view, the (dynamic) transition below $1 \mathrm{~K}$ behaves significantly different as compared to the spin-glass transitions observed in disordered $\operatorname{Li}_{1-x} \mathrm{Zn}_{x} \mathrm{~V}_{2} \mathrm{O}_{4}$ with $\mathrm{Zn}$ concentrations $x>0.05$ [15]. Instead we believe to have observed singlet formation (or another complex non-Néel state) in topologically frustrated $\mathrm{LiV}_{2} \mathrm{O}_{4}$ and further experiments to elucidate the ground state properties are highly needed. 
This work has partly been supported by the Sonderforschungsbereich 484 of the Deutsche Forschungsgemeinschaft and the BMBF under the contract number EKM 13N6917/0.

[1] D. C. Johnston, Physica B 281-282, 21 (2000)

[2] S. Kondo et al., Phys. Rev. Lett. 78, 3729 (1997)

[3] A. V. Mahajan et al., Phys. Rev. B 57, 8890 (1998)

[4] O. Chmaissem et al., Phys. Rev. Lett. 79, 4866 (1997)

[5] A. Krimmel et al., Phys. Rev. Lett. 82, 2919 (1999)

[6] C. M. Varma, Phys. Rev. B 60, R6973 (1999)

[7] V. I. Anisimov et al., Phys. Rev. Lett. 83, 364 (1999)

[8] V. Eyert et al., Europhys. Lett. 46, 762 (1999)

[9] J. Matsuno, A. Fujimori, and L. F. Mattheiss, Phys. Rev. B 60, 1607 (1999)

[10] D. J. Singh et al., Phys. Rev. B 60, 16359 (1999)

[11] a review is given in: T. Moriya, J. Magn. Magn. Mater. 14, 1 (1979)

[12] N. Fujiwara, Y. Ueda, and H. Yasuoka, Physica B 237-238, 59 (1997); N. Fujiwara, H. Yasuoka, and Y. Ueda, Phys. Rev. B 57, 3539 (1998); N. Fujiwara, H. Yasuoka, and Y. Ueda, Phys. Rev. B 59, 6294 (1999)

[13] H. Nakamura et al., J. Phys.: Condens. Matter 9, 4701 (1997)

[14] R. Ballou, E. Lelièvre-Berna, and B. Fåk, Phys. Rev. Lett. 76, 2125 (1996)

[15] W. Trinkl et al., Phys. Rev. B, to be published

[16] T. Moriya, J. Phys. Soc. Jpn. 16, 641 (1956); A. Narath and H. T. Weaver, Phys. Rev. 175, 373 (1968)

[17] O.O. Bernal et al., Phys. Rev. Lett. 75, 2023 (1995)

[18] D.L. Cox, N.E. Bickers, and J.W. Wilkins, J. Appl. Phys. 57, 3166 (1985)

[19] K. Asayama, Y. Kitaoka, and Y. Kohori, J. Magn. Magn. Mater. 76\&77, 449 (1988)

[20] P. Carreta, M. Corti, and A. Rigamonti, Phys. Rev. B 48, 3433 (1993); M. Corti et al., Phys. Rev. B 56, 11056 (1997)

[21] J. Merrin et al., J. Magn. Magn. Mater. 177-181, 799 (1998)

[22] K. Ishida et al., Phys. Rev. Lett. 82, 5353 (1999)

[23] Y. Okuno and K. Miyake, J. Phys. Soc. Jpn. 67, 3342 (1998)

[24] P. Chandra and P. Coleman in Les Houches 1991 Strongly Interacting Fermions and High Tc Superconductivity (Elsevier Science B.V., Amsterdam, North-Holland, 1995)

[25] J. Villain, Z. Physik B 33, 31 (1979)

FIG. 1. a) ${ }^{7} \mathrm{Li}$ Knight shift $K$ vs. temperature $T$ in $\mathrm{LiV}_{2} \mathrm{O}_{4}$ at two measuring frequencies/external fields, respectively: (o) $17.3 \mathrm{MHz} / 10 \mathrm{kOe}$ and $(\square) 72.7 \mathrm{MHz} / 44 \mathrm{kOe}$. b) ${ }^{7} \mathrm{Li}$ line width $\delta$ vs. temperature $T$. Inset: ${ }^{7}$ Li-NMR intesity multiplied by temperature and normalized $I \times T$ at $T=1 \mathrm{~K}$.

FIG. 2. Semi-logarithmic representation of the ${ }^{7} \mathrm{Li}$ spin-lattice relaxation rate $1 / T_{1}$ vs. temperature $T$ in $\mathrm{LiV}_{2} \mathrm{O}_{4}$ at two measuring frequencies/external fields, respectively: (o) $17.3 \mathrm{MHz} / 10 \mathrm{kOe}$ and ( $\square$ ) $72.7 \mathrm{MHz} / 44 \mathrm{kOe}$. The solid line indicates a Korringa relation which was found by Kondo et al. []

FIG. 3. a) ${ }^{7} \mathrm{Li}$ spin-lattice relaxation rate $1 / T_{1}$ vs. temperature $T$ in $\mathrm{LiV}_{2} \mathrm{O}_{4}$. b) Model calculations with one unique set of parameters as described in the text $\left(a=2.54 \mathrm{~s}^{-1} \mathrm{~K}^{-1}, b=5.34 \cdot 10^{11} \mathrm{~s}^{-2}, \Gamma_{0}=1.68 \mathrm{GHz}\right.$, and $\left.\Delta=1.47 \mathrm{~K}\right)$. c) Arrhenius plot of the spin-lattice relaxation rate $1 / T_{1}$. The solid lines indicate an activated behavior using a spin gap $\Delta_{S}=0.6 \mathrm{~K}$. 


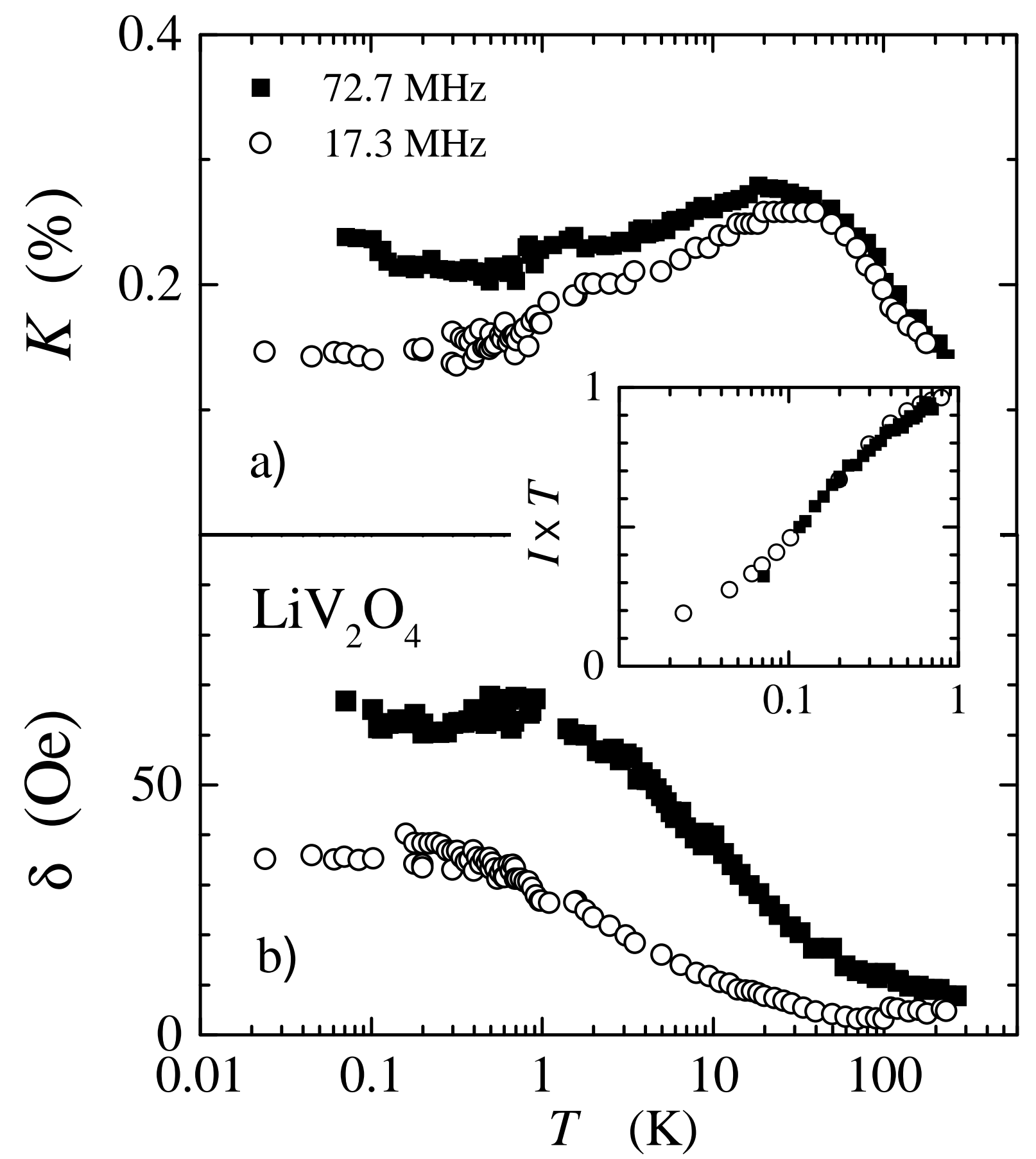




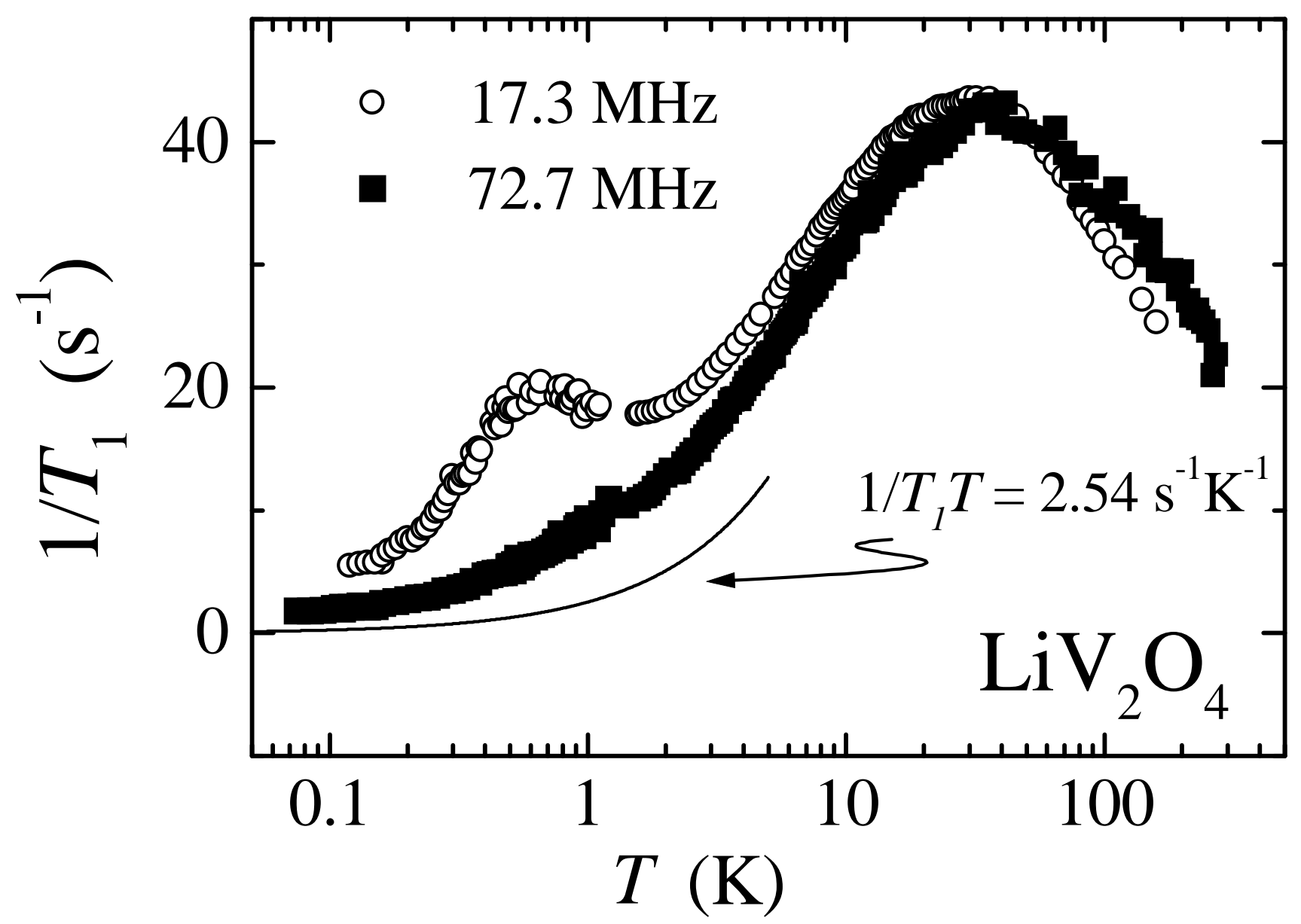




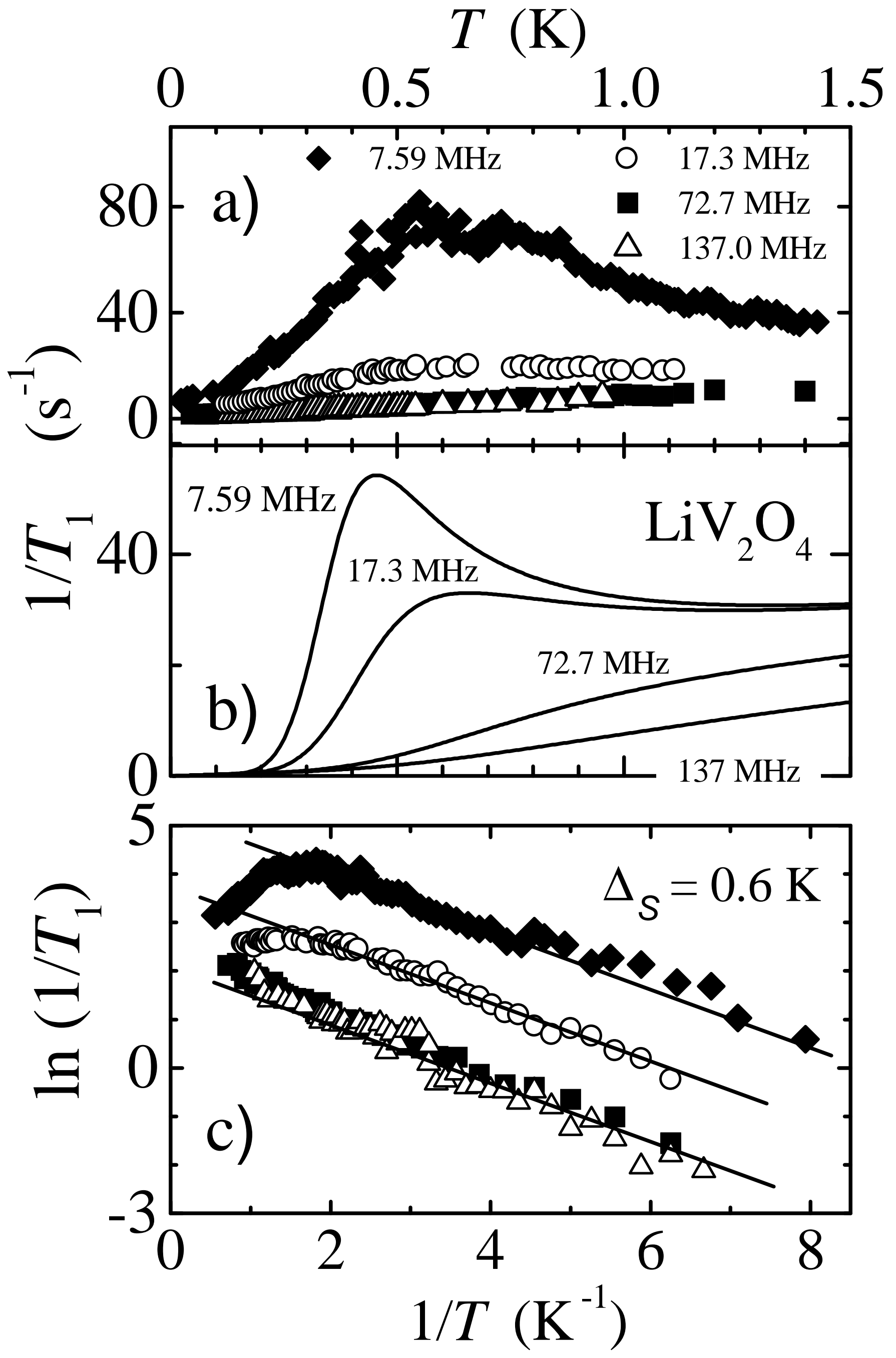

